\title{
sciendo
}

Int. J. of Applied Mechanics and Engineering, 2018, vol.23, No.4, pp.963-976

DOI: $10.2478 /$ ijame-2018-0054

\section{DOUBLE-DIFFUSIVE CONVECTION OF SYNOVIAL (COUPLE-STRESS) FLUID IN THE PRESENCE OF HALL CURRENT THROUGH A POROUS MEDIUM}

\author{
M. SINGH \\ Department of Mathematics, Govt PG College Seema (Rohru) \\ Distt Shimla (H.P)-171207, INDIA \\ E-mail: mahinder_singh91@rediffmail.in; drmsmath78@gmail.com
}

\begin{abstract}
An investigation made on the effect of Hall currents on double-diffusive convection of a compressible synovial (couple-stress) fluid in the presence of a horizontal magnetic field through a porous layer is considered. The analysis is carried out within the framework of linear stability theory and normal mode technique. A dispersion relation governing the effects of viscoelasticity, compressibility, magnetic field and porous layer is derived. For the stationary convection, a synovial fluid behaves like an ordinary Newtonian fluid due to the vanishing of the viscoelastic parameter. The stable-solute gradient, compressibility, and magnetic field have postponed the onset of convection, whereas Hall currents and medium permeability have not postponed the onset of convection, moreover, a synovial fluid has a dual character in the presence of Hall currents, whereas in the absence of Hall current in synovial fluid have postponed the onset of convection, which is in contrast in case of thermal convection couple-stress fluid with same effects. These analytic results are confirmed numerically and the effects of various parameters are depicted graphically. It has been observed that oscillatory modes are introduced due to the presence of viscoelasticity, magnetic field, porous medium and Hall currents which were non- existent in their absence. The sufficient conditions for the non-existence of overstability are also obtained.
\end{abstract}

Key words: compressibility, synovial fluid, double- diffusive convection, porous medium and Hall current.

Mathematical Classification (AMSC2010): 76A05, 76A10, 76D50, 76E06, 76E15, 76E07, 76E17, 76E19, 76N20, 76U05, 76W05, 80A20.

\section{Introduction}

The derivation of the basic equations of a layer of a fluid heated and soluted from below in a porous medium, using Boussinesq approximation, has been given in a treatise by Joseph [1]. The study of a layer of a fluid heated and soluted from below in a porous medium with Hall currents is motivated both by its theoretical and practical applications in engineering. Hall currents are effects whereby a conductor carrying an electric current perpendicular to an applied magnetic field develops a voltage gradient which is transverse to both the current and the magnetic field.

A normal synovial fluid is clear, pale yellow, viscid, and does not clot. Studies of mammalian synovial fluids have found considerable similarities among species, although notable differences do exist. The majority of investigative work determining the composition of a synovial fluid has been performed on the bovine synovial fluid mainly because large quantities of it are available. A synovial fluid is a plasma dialysate modified by constituents secreted by the joint tissues. The major difference between the synovial fluid and other body fluids derived from plasma is its high content of hyaluronic acid (mucin). The exact source of the hyaluronic acid has been the subject of debate. It is generally assumed, however, that both fibroblasts beneath the synovial membrane intima and synovial membrane-lining cells produce this mucopolysaccharide constituent of the synovial fluid. The synovial fluid is believed to have two main functions: to aid in the nutrition of articular cartilage by acting as a transport medium for nutritional 
substances, such as glucose, and to aid in the mechanical function of joints by lubrication of the articulating surfaces.

A couple-stress fluid is used to study the mechanisms of lubrications of synovial joints. A human joint is a dynamically loaded bearing which has an articular cartilage as the bearing and the synovial fluid as the lubricant. When a fluid film is generated, squeeze-film action is capable of providing considerable protection to the cartilage surface. The shoulder, ankle, knee and hip joints are the loaded-bearing synovial joints of the human body and these joints have a low friction coefficient and negligible wear. Normally, the synovial fluid is a viscous, non-Newtonian fluid and is clear or yellowish. The normal volume of the synovial fluid obviously varies from joint to joint. In a dog, the average is $0.24 \mathrm{ml}$, where as the normal synovial fluid complement levels in humans are approximately $10 \%$ of the serum values. [2]. Many theories based on extensive investigations of the physical properties and abilities of the synovial fluid to act as a lubricant have been presented to explain the mechanisms of joint lubrication. It appears that the low frictional resistance to join motion is due to a combination of mechanisms. Each mechanism complements the others and depends on the tissues involved and the load imparted to the joint [3].

The theory of couple-stress fluids initiated by Stokes [4], is a generalization of the classical theory of viscous fluids; an excellent introduction to this theory is available in the monograph "Theories of Fluids with Microstructure-An Introduction" written by Stokes [5] himself. The synovial fluid has been modeled as a couple-stress fluid in human joints by Walicki and Walicka [6].

In recent years, instability in a couple-stress fluid saturated porous layer heated and soluted from below has been investigated. The investigations included external constraints such as suspended particles, magnetic field and/or rotation. Sunil et al. [7] considered the effect of suspended particles on the stability of a couple-stress fluid layer heated and soluted from below in a porous medium. Malashetty et al. [8] studied the Soret effect on double-diffusive convection in a couple-stress liquid using both linear and non-linear analysis. In another study, Malashetty et al. $[9,10]$ analyzed the double-diffusive instability of a couplestress fluid saturated a porous layer. Singh and Kumar [11,12] studied rotatory thermosolutal convection in a couple - stress fluid and magneto-thermosolutal convection in a compressible couple- stress fluid. Singh and Kumar [13] studied magneto and rotatory thermosolutal convection through a porous medium. The effect of Hall currents was investigated by Sharma and Rani [14], Sunil et al. [15], Singh [16], as well as Jaimal et al. [17].

During the survey, it has been noticed that the Hall current effects are completely neglected in the studies of couple-stress fluids. Keeping in mind the importance of couple-stress fluids, convection in fluid layer soluted and heated from below through porous medium with Hall currents effects, the present paper attempts to study the effect of Hall current on thermosolutal convection of a couple-stress fluid through porous medium.

\section{Formulation of the problems}

Consider an infinite layer of an compressible finitely conducting (electrically and thermally) couplestress fluid through porous medium, heated and soluted from below, confined between two horizontal planes situated at $z=0$ and $z=d$, acted upon by a uniform horizontal magnetic field $H(H, 0,0)$, gravity field $g(0,0,-g)$. The fluid layer is heated from below such that a steady adverse temperature gradient $\beta=\frac{T_{0}-T_{1}}{d}$ , where $T_{0}$ and $T_{1}$ are the constant temperatures of the lower and upper boundaries with $T_{0}>T_{1}$ are maintained. 


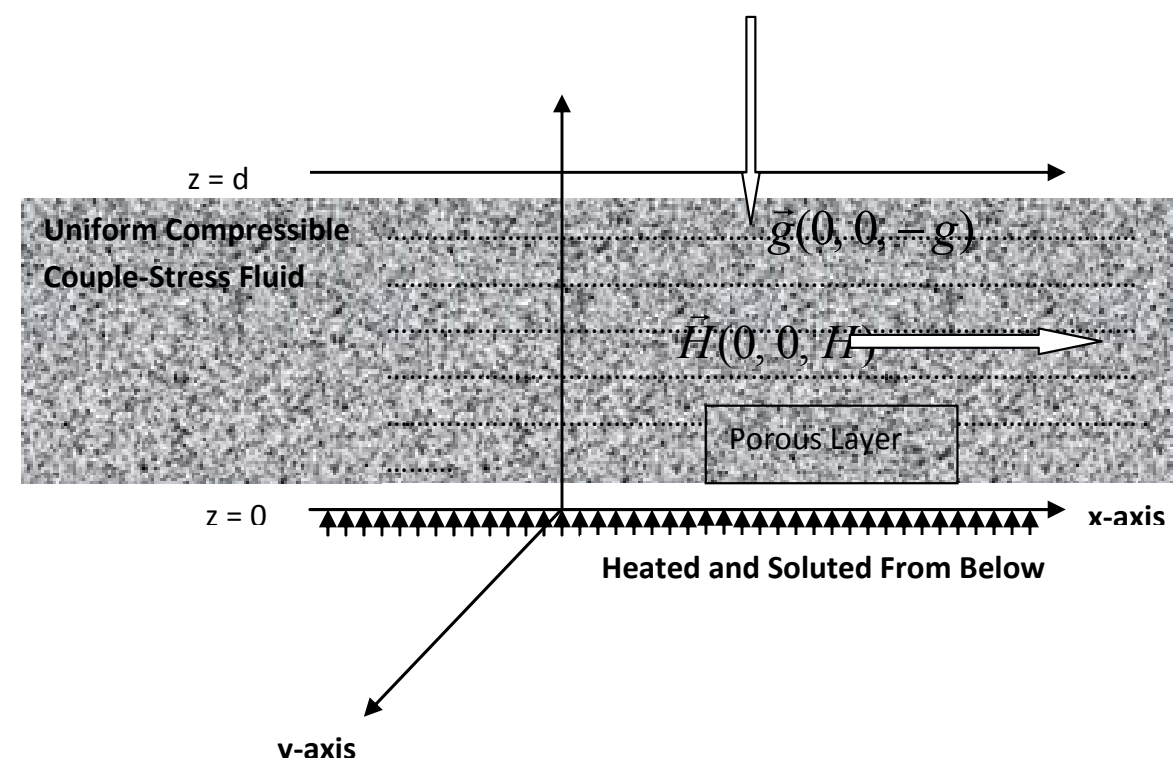

Fig.1. Geometrical representation of uniform compressible couple-stress fluid.

Since the non-linear theories attempt to allow for the finite amplitudes of the perturbations, we suppose that various physical variables describing the flow suffer small (infinitesimal) increments and, as a consequence, we neglect all product and power (higher than the first), of the increments and retain only terms that are linear and the linear stabilizing theory, for mathematical simplicity, is applied. Then, the linearized hydromagnetic equations relevant to the problem are

$$
\begin{aligned}
& \frac{1}{\epsilon}\left[\frac{\partial q}{\partial t}+\frac{1}{\epsilon}(q . \nabla) q\right]=-\frac{1}{\rho_{0}} \nabla p+g\left(1+\frac{\delta \rho}{\rho_{0}}\right)-\frac{1}{k_{l}}\left(v-\frac{\mu^{\prime}}{\rho_{0}} \nabla^{2}\right) q+\frac{\mu_{e}}{4 \pi \rho_{0}}(\nabla \times H) \times H, \\
& \nabla . q=0 \\
& E \frac{\partial T}{\partial t}+(q \cdot \nabla) T=k \nabla^{2} T, \\
& E^{\prime} \frac{\partial C}{\partial t}+(q \cdot \nabla) C=k^{\prime} \nabla^{2} C,
\end{aligned}
$$

Maxwell's equation yields

$$
\begin{aligned}
& \nabla . H=0, \\
& \in \frac{d H}{d t}=(H . \nabla) q+\in \eta \nabla^{2} H-\frac{c \in}{4 \pi N e} \nabla \times[(\nabla \times H) \times H]
\end{aligned}
$$

where $E=\in+(1-\epsilon)\left(\rho_{s} c_{s} / \rho c\right)$, here, $\rho, c$ and $\rho_{s}, c_{s}$ stand for the density and specific heat of the fluid and solid ( porous matrix) material, respectively; $v$, is the kinematic viscosity. The equation of state (Veronis, [18]) is

$$
\rho=\rho_{0}\left[1-\alpha\left(T-T_{0}\right)+\alpha^{\prime}\left(C-C_{0}\right)\right]
$$


where, the suffix zero refers to the values at the reference level $z=0$ and so the change in density $\delta \rho$, caused by perturbations $\theta$ and $\gamma$ in temperature and concentration is given by

$$
\delta \rho=-\rho_{0}\left(\alpha \theta-\alpha^{\prime} \gamma\right)
$$

The equation of state Eq.(2.7), contains a thermal coefficient of expansion $\alpha$ and an analogous solvent coefficient $\alpha^{\prime}$. Here $E^{\prime}$ is the solute parameter analogous to $E$. The steady state solution is

$$
\boldsymbol{q}(=0,0,0), \quad \boldsymbol{H}(=H, 0,0), \quad T=-\beta z+T_{0}, \quad C=-\beta z+C_{0}, \quad \rho=\rho_{0}\left(1+\alpha \beta z-\alpha^{\prime} \beta^{\prime} z\right)
$$

where $\beta$ and $\beta^{\prime}$ are both positive. Here, we consider the case, in which both boundaries are free as well as perfect conductors of heat. Since the boundaries are maintained at constant temperature, the perturbation in the temperature is zero at the boundaries and concentration at a boundary is kept fixed, therefore the appropriate boundary conditions are

$$
\begin{aligned}
& W=D^{2} W=X=D Z=0=\Theta=\Gamma \quad \text { at } \quad z=0 \text { and } 1 . \\
& W=W_{0} \sin \pi z .
\end{aligned}
$$

After using the boundary conditions, Eqs (2.1)-(2.6) become

$$
\begin{aligned}
& \frac{1}{\in} \frac{\partial q}{\partial t}=-\frac{1}{\rho_{0}}(\nabla . \delta p)-g\left(\alpha \theta-\alpha^{\prime} \gamma\right)-\frac{1}{k_{l}}\left(v-\frac{\mu^{\prime}}{\rho_{0}} \nabla^{2}\right) q+\frac{\mu_{e}}{4 \pi \rho_{0}}(\nabla \times h) \times H, \\
& \nabla . q=0 \\
& E \frac{\partial \theta}{\partial t}=\left(\beta-\frac{g}{c_{p}}\right) w+k \nabla^{2} \theta, \\
& E^{\prime} \frac{\partial \gamma}{\partial t}=\beta^{\prime} w+k^{\prime} \nabla^{2} \gamma, \\
& \in \frac{\partial h}{\partial t}=(H . \nabla) q+\in \eta \nabla^{2} h-\frac{c \in}{4 \pi N e} \nabla \times[(\nabla \times h) \times H], \\
& \nabla . h=0 .
\end{aligned}
$$

\section{Dispersion relation}

Analyzing the disturbances into normal modes, we assume that the perturbation quantities are of the form

$$
\left(w, h_{z}, \theta, \gamma, \varsigma, \xi\right)=[W(z), K(z), \Theta(z), \Gamma(z), X(z)] \exp \cdot\left[i k_{x} x+i k_{y} y+n t\right]
$$

where $k_{x}, k_{y}$ are the wave numbers along the $x$-axis and $y$-axis, respectively; $\varsigma=\frac{\partial v}{\partial x}-\frac{\partial u}{\partial y}$, and $\xi=\frac{\partial h_{y}}{\partial x}-\frac{\partial h_{x}}{\partial y}$, respectively. Using expression (3.1), Eqs (2.11)-(2.16), in a non dimensional form become 


$$
\begin{aligned}
& \left(D^{2}-a^{2}\right)\left[\frac{\sigma}{\epsilon}+\frac{1}{P_{l}}-\frac{F}{P_{l}}\left(D^{2}-a^{2}\right)\right] W-\frac{\mu_{e} H d}{4 \pi \rho_{0} v}\left(D^{2}-a^{2}\right) D K+\frac{g a^{2} d^{2}}{v}\left(\alpha \Theta-\alpha^{\prime} \Gamma\right)=0, \\
& {\left[\frac{\sigma}{\epsilon}+\frac{1}{P_{l}}-\frac{F}{P_{l}}\left(D^{2}-a^{2}\right)\right] Z=\frac{\mu_{e} H d}{4 \pi \rho_{0} v} D X} \\
& \left(D^{2}-a^{2}-p_{2} \sigma\right) K=-\frac{H d}{\eta \in} D W+\frac{c H d}{4 \pi N e \eta} D X \\
& \left(D^{2}-a^{2}-p_{2} \sigma\right) X=-\left(\frac{H d}{\eta \epsilon}\right) D Z-\frac{c H}{4 \pi N e \eta d}\left(D^{2}-a^{2}\right) D K \\
& \left(D^{2}-a^{2}-E p_{1} \sigma\right) \Theta=-\left(\frac{1}{G-1}\right) \frac{g d^{2}}{k C_{p}} W \\
& \left(D^{2}-a^{2}-E^{\prime} q \sigma\right) \Gamma=-\frac{\beta^{\prime} d^{2}}{k^{\prime}} W
\end{aligned}
$$

where we have to put $a=k d, \sigma=n d^{2} / v, x / d=x^{*}, y / d=y^{*}, z / d=z^{*}$ and $D=d / d z^{*}$. Here $p_{1}=v / \kappa$, $p_{2}=v / \eta, q=v / \kappa^{\prime}, P_{l}=k_{1} / d^{2}$, and $F=\mu^{\prime} / \rho_{0} d^{2} v$. We shall suppress the star $(*)$, for convenience hereafter. Eliminating $\Theta, \Gamma$ and $K$ in Eqs (3.2)-(3.7) and using the proper solution $W=W_{0} \sin \pi z$, we obtain the dispersion relation as

$$
\begin{aligned}
& R_{l}=\left(\frac{G}{G-1}\right)\left[( \frac { 1 + x } { x } ) \left[\left\{\left(\frac{i \sigma_{1}}{\in}+\frac{1}{P}+\frac{\pi^{2} F(1+x)}{P}\right)\left(1+x+i E p_{l} \sigma_{l}\right)\right\}+\right.\right. \\
& \left.+\frac{Q_{l}\left(1+x+i E p_{1} \sigma_{1}\right)\left\{\left(\frac{i \sigma_{1}}{\epsilon}+\frac{1}{P}+\frac{\pi^{2} F(1+x)}{P}\right)\left(1+x+i p_{2} \sigma_{l}\right)+Q_{1}\right\}}{\left\{\left(\frac{i \sigma_{1}}{\epsilon}+\frac{1}{P}+\frac{\pi^{2} F(1+x)}{P}\right)\left(1+x+i p_{2} \sigma_{l}\right)^{2}+Q_{l}\left(1+x+i p_{2} \sigma_{l}\right)+M(1+x)\left(\frac{i \sigma_{1}}{\epsilon}+\frac{1}{P}+\frac{\pi^{2} F(1+x)}{P}\right)\right\}}\right]+ \\
& \left.+S_{1} \frac{\left(1+x+i E p_{1} \sigma_{1}\right)}{\left(1+x+i E^{\prime} q \sigma_{1}\right)}\right]
\end{aligned}
$$

where

$$
\begin{aligned}
& R_{l}=\frac{g \alpha \beta d^{4}}{v \kappa \pi^{4}}, \quad S_{l}=\frac{g \alpha^{\prime} \beta^{\prime} d^{4}}{v \kappa^{\prime} \pi^{4}}, \quad Q_{l}=\frac{\mu_{e} H^{2} d^{2}}{4 \pi \rho_{0} \nu \eta \pi^{2}}, \quad P=\pi^{2} P_{l}, \\
& M=\left(\frac{c H}{4 \pi N e \eta}\right)^{2}, \quad x=a^{2} / \pi^{2} \text { and } i \sigma_{1}=\sigma / \pi^{2} .
\end{aligned}
$$




\section{Stationary convection}

For the stationary convection, put $i \sigma_{1}=0$, in Eq.(3.8), we get

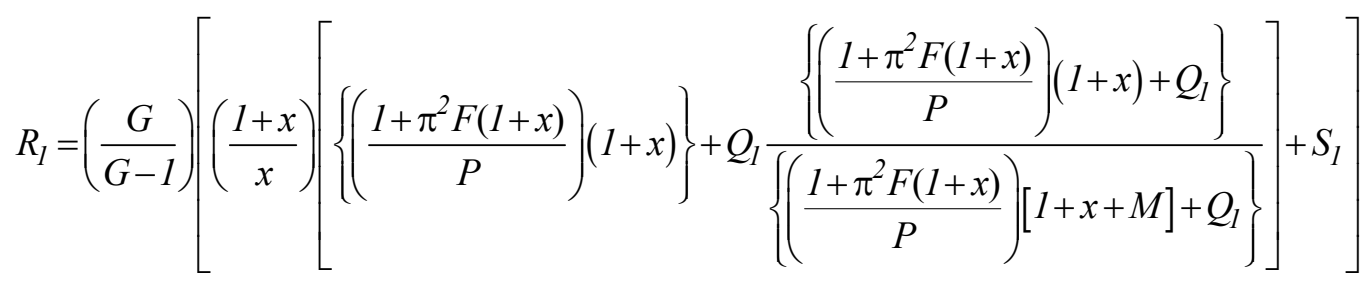

The above relation is the modified result of the Rayleigh number $R_{l}$, as a function of the parameters $Q_{1}, S_{1}, M, P$ and $F$, and dimensionless wave number $x$. For fixed (accounting for the compressibility effects), $Q_{1}, S_{1}, M, P$ and $F$, we find that

$$
\bar{R}_{c}=\left(\frac{G}{G-1}\right) R_{c}
$$

where $R_{c}$ and $\overline{R_{c}}$ denote the critical Rayleigh numbers in the absence and presence of compressibility. The cases $G<1$ and $G=1$ correspond to negative and infinite value of the Rayleigh number which are not relevant in the present study. Since the critical Rayleigh number is positive and finite, so $G>1$ has the effect of compressibility postponed the onset of thermosolutal convection.

For the case of stationary convection, to study the effect of Hall currents, the magnetic field, synovial fluid, stable solute gradient and medium permeability, we examine the nature of $\frac{d R_{1}}{d Q_{1}}, \frac{d R_{1}}{d S_{1}}, \frac{d R_{1}}{d M}$, $\frac{d R_{l}}{d P}$ and $\frac{d R_{1}}{d F}$. Equation (4.1) yields

$$
\frac{d R_{1}}{d Q_{1}}=-\left(\frac{G}{G-1}\right)\left(\frac{1+x}{x}\right) \frac{\left[\left(\frac{1+\pi^{2} F(1+x)}{P}\right)^{2}(1+x+M)(1+x)+2 Q_{l}\left(\frac{1+\pi^{2} F(1+x)}{P}\right)(1+x+M)+Q_{l}^{2}\right]}{\left[\left(\frac{1+\pi^{2} F(1+x)}{P}\right)(1+x+M)+Q_{l}\right]^{2}},
$$

which is negative and shows that magnetic field has not postponed the onset of double-diffusive convection in the compressible synovial fluid in a porous medium.

Expression

$$
\frac{d R_{1}}{d S_{1}}=\left(\frac{G}{G-1}\right)
$$

is positive and shows that the stable solute gradient has postponed the onset of convection in the compressible synovial (couple-stress) fluid.

If expression 


$$
\frac{d R_{1}}{d M}=-\left(\frac{G}{G-1}\right)\left(\frac{1+x}{x}\right) \frac{\left[Q_{1}\left\{\left(\frac{1+\pi^{2} F(1+x)}{P}(1+x)\right)+Q_{1}\right\}\left(\frac{1+\pi^{2} F(1+x)}{P}\right)\right]}{\left[\left(\frac{1+\pi^{2} F(1+x)}{P}\right)(1+x+M)+Q_{1}\right]^{2}}
$$

is negative, therefore Hall currents have not postponed the onset of convection in the double-diffusive compressible synovial fluid in a porous medium.

If expression

$$
\begin{aligned}
& \frac{d R_{1}}{d P}=-\left(\frac{G}{G-1}\right)\left(\frac{1+x}{x}\right)\left(\frac{1+\pi^{2} F(1+x)}{P^{2}}\right) \times \\
& \times \frac{\left[\left(\frac{1+\pi^{2} F(1+x)}{P}\right)(1+x)(1+x+M)\left\{\left(\frac{1+\pi^{2} F(1+x)}{P}\right)(1+x+M)+2 Q_{1}\right\}+Q_{1}^{2}(1+x-M)\right]}{\left[\left(\frac{1+\pi^{2} F(1+x)}{P}\right)(1+x+M)+Q_{1}\right]^{2}},
\end{aligned}
$$

is negative, therefore medium permeability has not postponed the onset of convection.

Expression

$$
\frac{d R_{l}}{d F}=\left(\frac{G}{G-1}\right) \frac{\pi^{2}(1+x)^{2}}{x P} \frac{\left[\left(\frac{1+\pi^{2} F(1+x)}{P}\right)(1+x)(1+x+M)\left\{\left(\frac{1+\pi^{2} F(1+x)}{P}\right)(1+x+M)+2 Q_{l}\right\}+Q_{l}^{2}(1+x-M)\right]}{\left[\left(\frac{1+\pi^{2} F(1+x)}{P}\right)(1+x+M)+Q_{l}\right]^{2}}
$$

shows that the synovial fluid has postponed or has not postponed the onset of convection according as $(1+x)>M$ or $(1+x)<M$. It is to be noted that in the absence of Hall currents, Eq.(4.7), gives

$$
\frac{d R_{1}}{d F}=\left(\frac{G}{G-1}\right) \frac{\pi^{2}(1+x)^{2}}{x P} \frac{\left[\left(\frac{1+\pi^{2} F(1+x)}{P}\right)(1+x)^{2}\left\{\left(\frac{1+\pi^{2} F(1+x)}{P}\right)(1+x)+2 Q_{1}\right\}+Q_{1}^{2}(1+x)\right]}{\left[\left(\frac{1+\pi^{2} F(1+x)}{P}\right)(1+x)+Q_{1}\right]^{2}},
$$

which is positive and shows that the synovial fluid has postponed the onset of convection. Thus the synovial fluid has a dual character, in the absence of Hall currents it has postponed (stabilized) the onset of convection, while in their presence it may or may not be postponed (stabilized).

The dispersion relation Eq.(4.1), is also analyzed numerically, for the various values of $M, P, F$, $Q_{1}$ and $S_{1}$. It is also evident from Figs 1-6 that the stable solute gradient, magnetic field, synovial fluid parameters have stabilizing effects on the system, where as Hall currents, medium permeability have destabilizing effects on the system. Figure 1 show the geometry of the problem which I have used. In Figs 26 , the Rayleigh number is analyzed with wave number and found that in Fig.2, as values of $Q_{1}$ increased, the 
values of Rayleigh number is increased, shows that magnetic field has stabilizing effect on the system. In Fig.3, as values of $S_{1}$ is increased, gives the same result as $Q_{1}$ and $F$ in Fig.6, shows that stable solute gradient as well as couple-stress parameters have stabilizing effects on the system. In Fig.4 as values of $M$ increased value of Rayleigh number decrease, shows that Hall current parameter have destabilizing effect on the system. In the Fig.5 as values of $P$ increased gives the same results as in case of Hall currents, shows that medium permeability have destabilizing effect on the system.

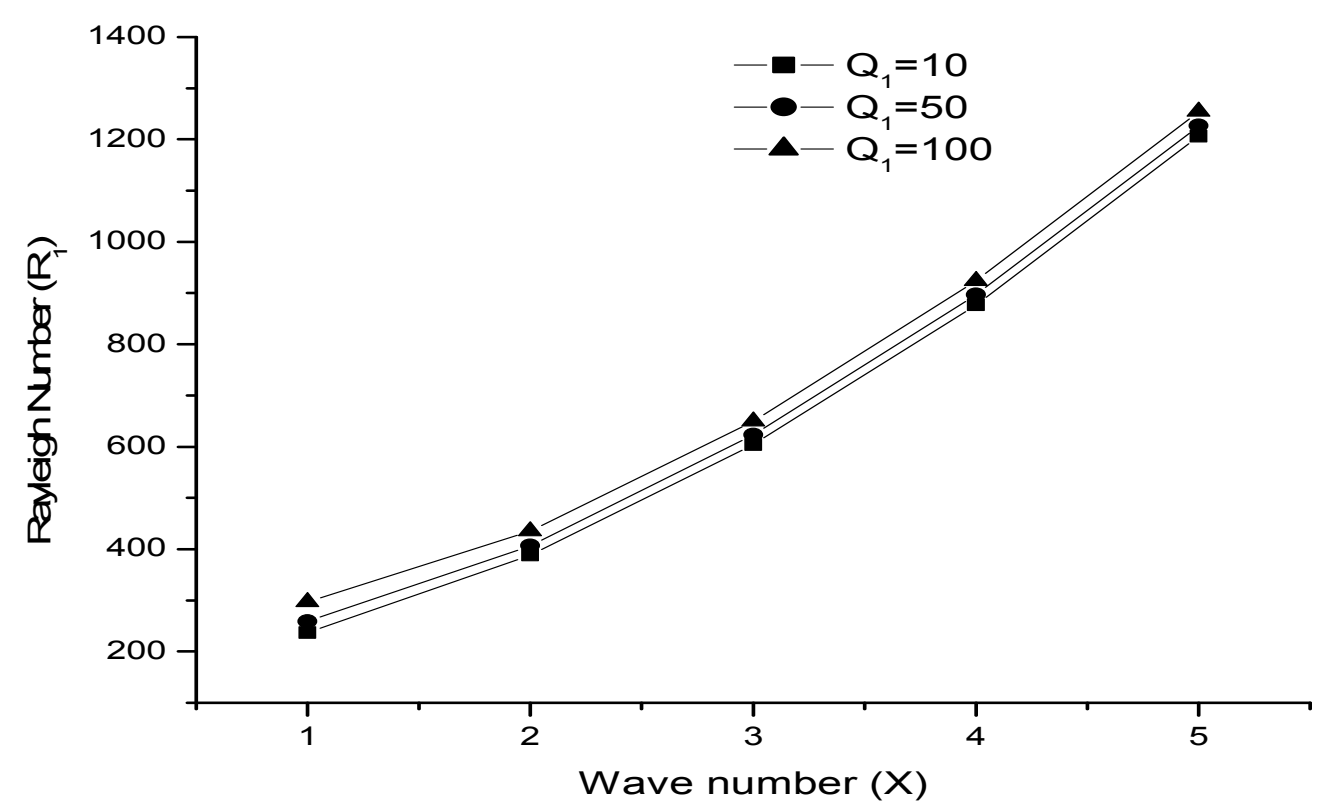

Fig.2. Variation of Rayleigh number $\left(\mathrm{R}_{1}\right)$, with wave number $X(=1-5)$, for $Q_{1}(=10,50,100)$, when $M=10$, $S_{I}=10, P=2, F=5, \pi=3.14$ and $G=9.8$.

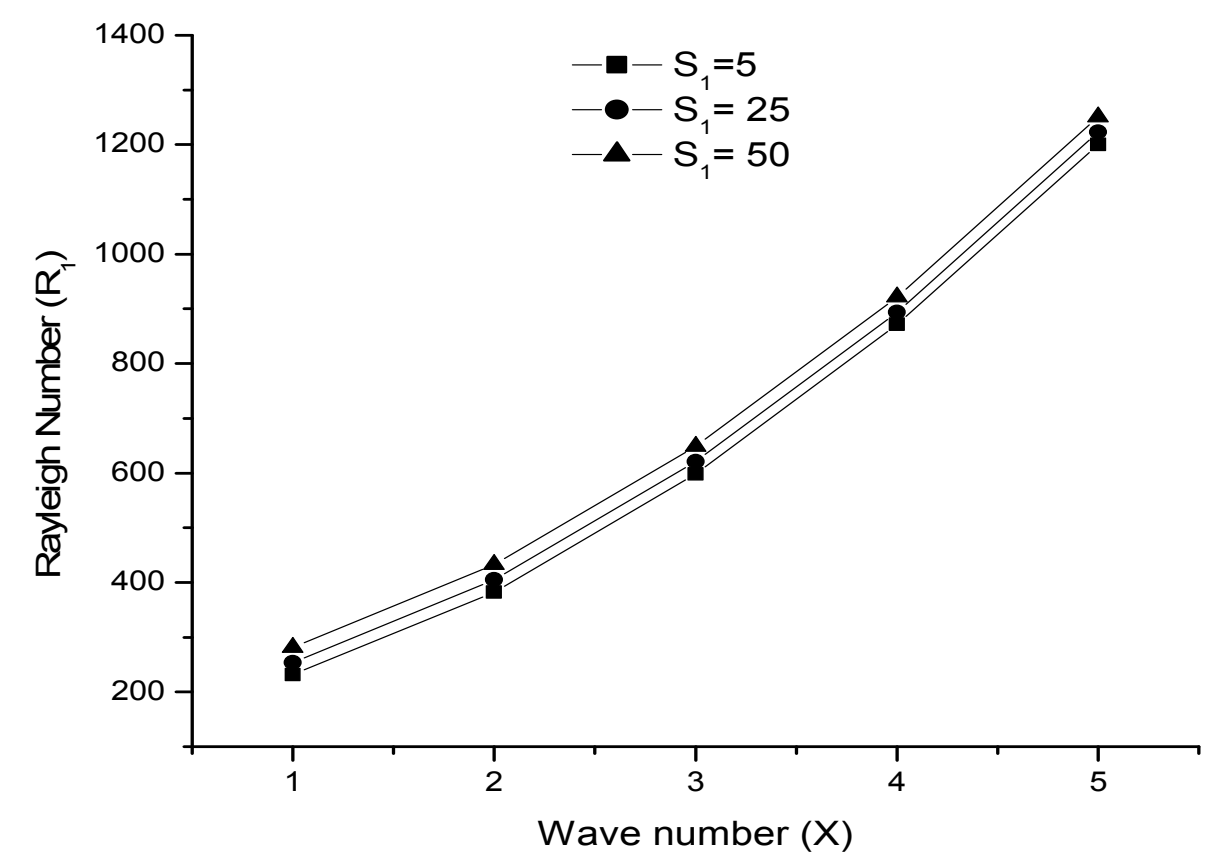

Fig.3. Variation of Rayleigh number $\left(\mathrm{R}_{1}\right)$, with wave number $X(=1-5)$, for $S_{I}(=05,25,50)$, when $M=10$, $P=02, Q_{I}=10, F=05, \pi=3.14$ and $G=9.8$. 


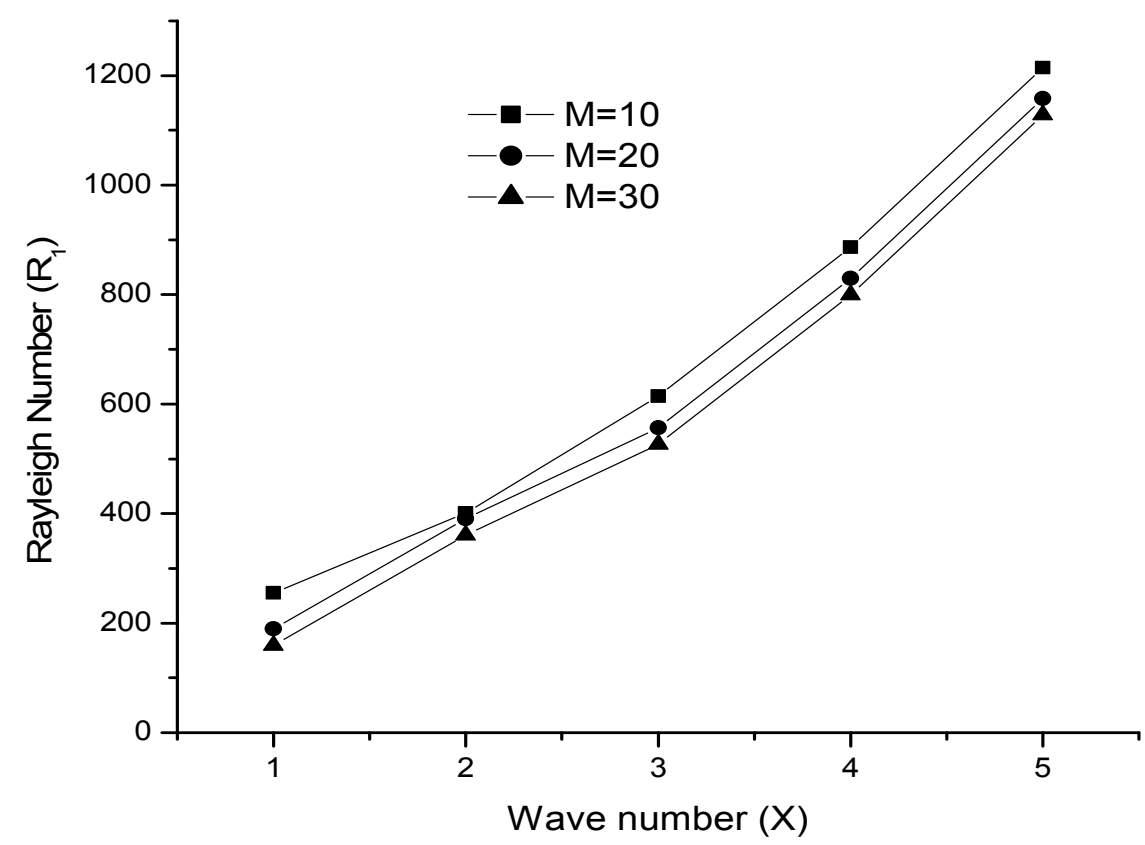

Fig.4. Variation of Rayleigh number $\left(\mathrm{R}_{1}\right)$, with wave number $X(=1-5)$, for $M(=10,20,30)$, when $S_{1}=10$, $P=2, Q_{1}=10, F=5, \pi=3.14$ and $G=9.8$.

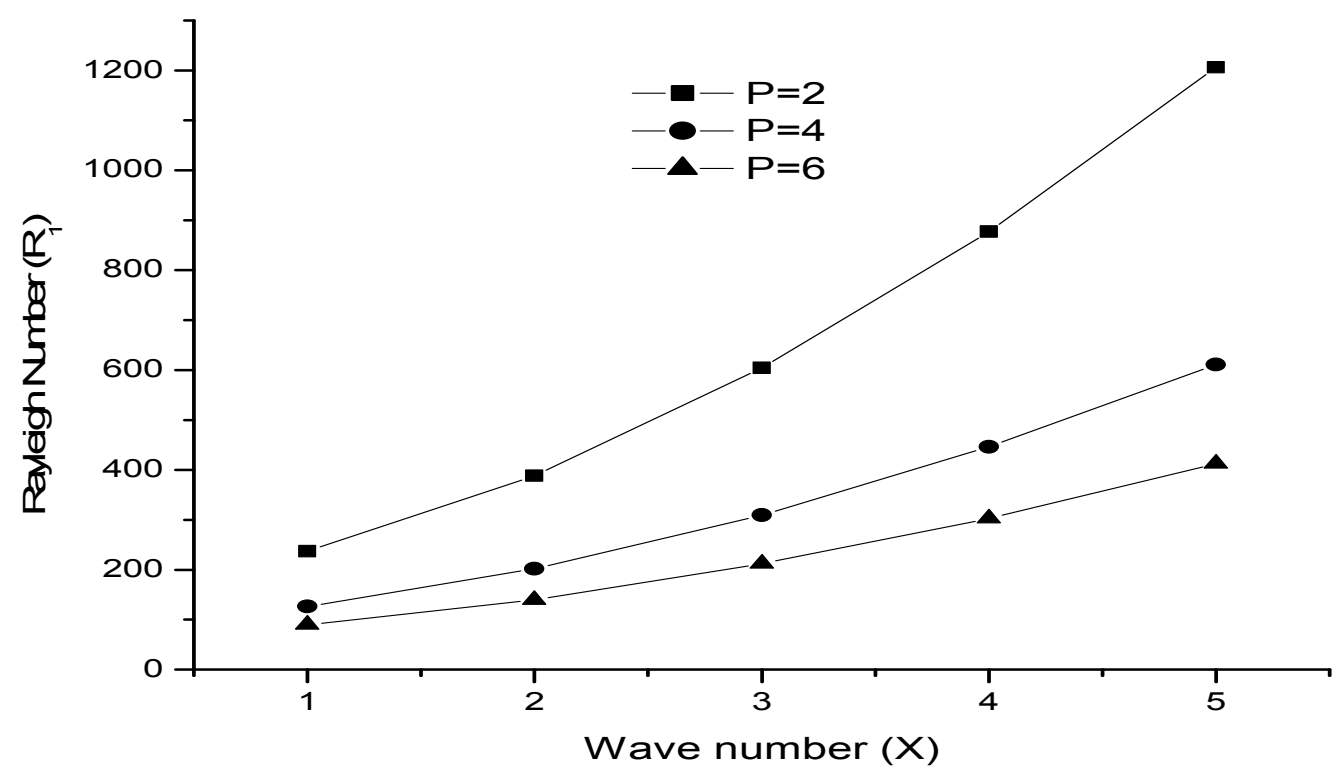

Fig.5. Variation of Rayleigh number $\left(\mathrm{R}_{1}\right)$, with wave number $X(=1-5)$, for $P(=2,4,6), S_{1}=10, M=10$, $Q_{l}=10, F=5, \pi=3.14$ and $G=9.8$. 


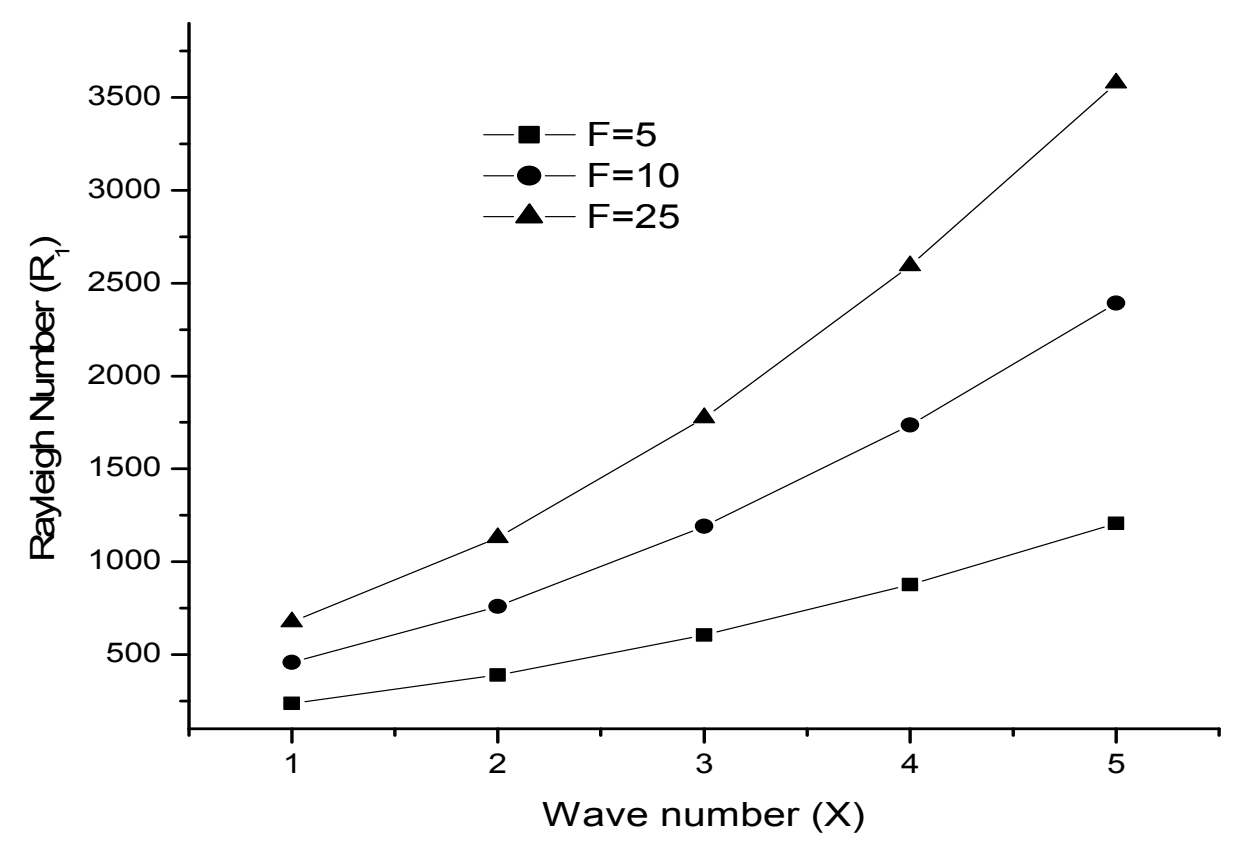

Fig.6. Variation of Rayleigh number $\left(\mathrm{R}_{1}\right)$, with wave number $X(=1-5)$, for $F(=5,10,15)$, when $Q_{1}=10$, $S_{1}=10, M=10, P=2, \pi=3.14$ and $G=9.8$.

\section{Stability of the system and oscillatory modes}

Multiplying Eq.(3.2) by $W^{*}$ (the complex conjugate of $W$ ), integrating over the range of $z$ and makings use of Eqs (3.3) to (3.7), together with boundary condition (2.10), we get

$$
\begin{aligned}
& F I_{1}+\left(1+P_{l} \frac{\sigma}{\epsilon}\right) I_{2}-\frac{g \alpha K a^{2}}{v \beta}\left(\frac{G}{G-1}\right) P_{l}\left[I_{3}+E p_{1} \sigma^{*} I_{4}\right]+\frac{\mu_{e} \in \eta}{4 \pi \rho_{0} v} P_{l}\left[I_{5}+p_{2} \sigma^{*} I_{6}\right]+ \\
& +\frac{\mu_{e} \in \eta d^{2}}{4 \pi \rho_{0} v} P_{l}\left[I_{9}+p_{2} \sigma I_{10}\right]+d^{2}\left[\left(1+P_{l} \frac{\sigma^{*}}{\epsilon}\right) I_{8}+F I_{7}\right]+\left(\frac{g \alpha^{\prime} k^{\prime} a^{2}}{v \beta^{\prime}}\right)\left[I_{11}+E^{\prime} q \sigma^{*} I_{12}\right]=0
\end{aligned}
$$

where integrals $I_{1}-I_{12}$, are positive definite. Putting $\sigma=\sigma_{r}+i \sigma_{i}$ and equating the real and imaginary parts of Eq.(5.1), we have

$$
\begin{aligned}
& \sigma_{r}\left[\frac{I_{2}}{\epsilon}-\frac{g \alpha G}{G-1} \frac{k a^{2}}{v \beta} E p_{1} I_{4}+\frac{\mu_{e} \in \eta}{4 \pi \rho_{0} \mathrm{v}} p_{2}\left(I_{6}+d^{2} I_{10}\right)+\frac{d^{2}}{\epsilon} I_{8}+\frac{g \alpha^{\prime} k^{\prime} a^{2}}{v \beta^{\prime}} E^{\prime} q I_{12}\right]= \\
& =-\left[\frac{F}{P_{l}} I_{1}+\frac{1}{P_{l}} I_{2}-\frac{G g \alpha k a^{2}}{(G-1) \mathrm{v} \beta} I_{3}+\frac{\mu_{e} \in \eta}{4 \pi \rho_{0} \mathrm{v}}\left(I_{5}+d^{2} I_{9}\right)+\frac{d^{2}}{P_{l}}\left(I_{8}+F I_{7}\right)+\frac{g \alpha^{\prime} k^{\prime} a^{2}}{v \beta^{\prime}} I_{11}\right], \\
& i \sigma_{i}\left[\frac{I_{2}}{\epsilon}+\frac{g \alpha k a^{2}}{v \beta} E p_{1} I_{4}-\frac{\mu_{e} \in \eta}{4 \pi \rho_{0} \mathrm{v}} p_{2}\left(I_{8}-d^{2} I_{12}\right)-\frac{d^{2}}{\epsilon} I_{10}+\frac{g \alpha k a^{2}}{v \beta} E p_{1} I_{4}-\frac{g \alpha^{\prime} k^{\prime} a^{2}}{v \beta^{\prime}} E^{\prime} q I_{12}\right]=0,
\end{aligned}
$$




$$
\begin{aligned}
& I_{1}=\int_{0}^{1}\left(\left|D^{2} W\right|^{2}+2 a^{2}|D W|^{2}+a^{4}|W|^{2}\right) d z, \quad I_{2}=\int_{0}^{1}\left(|D W|^{2}+a^{2}|W|^{2}\right) d z, \\
& I_{3}=\int_{0}^{1}\left(|D \Theta|^{2}+a^{2}|\Theta|^{2}\right) d z, \quad I_{4}=\int_{0}^{1}\left(|\Theta|^{2}\right) d z, \quad I_{5}=\int_{0}^{1}\left(\left|D^{2} K\right|^{2}+2 a^{2}|D K|^{2}+a^{4}|K|^{2}\right) d z, \\
& I_{6}=\int_{0}^{1}\left(|D K|^{2}+a^{2}|K|^{2}\right) d z, \quad I_{7}=\int_{0}^{1}\left(|D Z|^{2}+a^{2}|Z|^{2}\right) d z, \quad I_{8}=\int_{0}^{1}\left(|Z|^{2}\right) d z, \\
& I_{9}=\int_{0}^{1}\left(|D X|^{2}+a^{2}|X|^{2}\right) d z, \quad I_{10}=\int_{0}^{1}\left(|X|^{2}\right) d z, \\
& I_{11}=\int_{0}^{1}\left(|D \Gamma|^{2}+a^{2}|\Gamma|^{2}\right) d z, \quad I_{12}=\int_{0}^{1}\left(|\Gamma|^{2}\right) d z .
\end{aligned}
$$

In the absence of the solute gradient and magnetic field, Eqs (5.3) reduces to

$$
\left[\frac{I_{2}}{\epsilon}+\frac{g \alpha k a^{2}}{v \beta} E p_{1} I_{4}\right] \sigma_{i}=0 \text {. }
$$

It may be inferred from Eqs (5.2) and (5.3), that $\sigma_{i}$ may be positive or negative, which means that the system may be stable or unstable, while in the absence of the solute gradient and magnetic field, Eq.(5.5) predicts that $\sigma_{i}=0$ necessarily because all the terms in the brackets are positive definite, which implies that oscillatory modes are not allowed in the system.

\section{The case of overstability}

In the present section, we discuss the possibility whether instability may occur as overstability. Since for overstability, we wish to determine the critical Rayleigh number for the onset of instability (via a state of pure oscillations), it will suffice to find conditions for which Eq.(3.8) will admit solutions with $\sigma_{l}$ being real. Equating real and imaginary parts of Eq.(3.8), and eliminating $R_{1}$ between them, we obtain

$$
\begin{aligned}
& A_{4} C_{1}^{4}+A_{3} C_{1}^{3}+A_{2} C_{1}^{2}+A_{1} C_{1}+A_{0}=0 \\
& A_{4}=\frac{E^{\prime 2} q^{2} p_{2}{ }^{4}}{\epsilon^{2}}\left[\left(\frac{1}{\epsilon}+\frac{E p_{1} \pi^{2} F}{P}\right) b+\frac{E p_{1}}{P}\right],
\end{aligned}
$$




$$
\begin{aligned}
& A_{3}=\left[\left\{E^{\prime 2} q^{2}\left(\frac{1}{\epsilon}+\frac{E p_{1} \pi^{2} F}{P}\right)\left(\frac{p_{2}{ }^{2} \pi^{4} F^{2}}{P^{2}}+\frac{2}{\epsilon^{2}}\right) p_{2}{ }^{2}+\frac{p_{2}{ }^{4}}{\epsilon^{2}}\left(\frac{1}{\epsilon}+\frac{E p_{1} \pi^{2} F}{P}\right)\right\} b^{3}+\right. \\
& +\left\{2 E^{\prime 2} q^{2}\left(\frac{1}{\epsilon}+\frac{E p_{1} \pi^{2} F}{P}\right)\left(\frac{p_{2}{ }^{2} \pi^{2} F}{P}-\frac{M}{\epsilon^{2}}\right) p_{2}{ }^{2}+\frac{p_{2}{ }^{4} E p_{1}}{\epsilon^{2} P}+\frac{E^{\prime 2} q^{2} E p_{1}}{P}\left(\frac{p_{2}{ }^{2} \pi^{4} F^{2}}{P^{2}}+\frac{2}{\epsilon^{2}}\right) p_{2}{ }^{2}\right\} b^{2}+ \\
& +E^{\prime 2} q^{2}\left\{\left(\frac{1}{\epsilon}+\frac{E p_{1} \pi^{2} F}{P}\right)\left(\frac{p_{2}}{P^{2}}-\frac{2 Q_{1}}{\epsilon}\right) p_{2}{ }^{3}+\frac{2 E p_{1} p_{2}{ }^{2}}{P}\left(\frac{p_{2}{ }^{2} \pi^{2} F}{P^{2}}-\frac{M}{\epsilon^{2}}\right)+\left(\frac{p_{2}{ }^{2}}{\epsilon^{2}}\left(E p_{1}-p_{2}\right)\right)\right\} b+ \\
& \left.\left\{\frac{E^{\prime 2} q^{2} E p_{1}}{P}\left(\frac{p_{2}}{P^{2}}-\frac{2 Q_{1}}{\epsilon}\right) p_{2}{ }^{3}+\left(\frac{p_{2}{ }^{4}}{\epsilon^{2}} S_{1}\left(E p_{1}-E^{\prime} q\right)\right)(b-1)\right\}\right]
\end{aligned}
$$

where $b=1+x$. Other coefficients are positive very lengthy and do not effect the system. Here, $A_{4}$ is positive and $A_{3}$ is positive in Eq.(6.1) if

$$
E p_{1}>p_{2}, E p_{1}>E^{\prime} q, \frac{p_{2}{ }^{2} \pi^{2}}{P^{2}}>\frac{M}{\epsilon^{2}} \text { and } \frac{p_{2}}{P^{2}}>\frac{2 Q_{1}}{\epsilon}, k<E \eta, k<\frac{E k^{\prime}}{E^{\prime}}, \mathrm{v}>\frac{1}{\mu^{\prime}}\left(\frac{c H k_{1} \pi^{2}}{4 \in N e}\right)^{2}
$$

which imply that

$$
k<\min \left(E \eta, \frac{E k^{\prime}}{E^{\prime}}\right), \quad v>\max \left[\frac{1}{\mu^{\prime}}\left(\frac{c H k_{1} \pi^{2}}{4 \in N e}\right)^{2}, \sqrt{\frac{\mu_{e} \pi}{2 \rho_{0}}} \frac{k_{l} H}{\in d}\right],
$$

are sufficient conditions for the non-existence of overstability, the violation of which does not necessarily imply the occurrence of overstability.

\section{Concluding remarks}

Combined effects of various parameters, i.e. the stable solute gradient, magnetic field, Hall currents, medium permeability and compressibility, have been investigated in double-diffusive convection of a synovial (couple-stress) fluid. The concluding remarks are as follows:

(i): For stationary convection, a synovial(couple-stress) fluid behaves like an ordinary Newtonian fluid, due to the vanishing of the viscoelastic parameters.

(ii): From Eq.(4.2), it is clear that the effect of compressibility has onset of convection only for $G>1$.

(iii): The presence of the magnetic field (and therefore Hall currents), and medium permeability effects introduce oscillatory modes in the system, in the absence of Hall currents, oscillatory modes are not allowed in the system and the principle of exchange of stability is valid here.

(iv): In ordinary (stationary convection) of a synovial fluid, we found that the stable-solute gradient and magnetic field have postponed the onset of convection, whereas Hall currents and medium permeability have not postponed the onset of convection. A synovial fluid has postponed as well as has not postponed the onset of convection in the presence of Hall currents. But in the absence of Hall currents synovial fluid has only postponed the onset of convection so couple-stress has dual character.

(v): Results are depicted graphically here (i.e. Figs 2-6). We found that the stable-solute gradient, magnetic field, and synovial fluid have stabilizing effects on the system, whereas Hall currents and medium permeability have destabilizing effects on the system. 
(vi): The sufficient conditions for the occurrence of overstability are $k<\min \left(E \eta, \frac{E k^{\prime}}{E^{\prime}}\right)$, $v>\max \left[\frac{1}{\mu^{\prime}}\left(\frac{c H k_{1} \pi^{2}}{4 \in N e}\right)^{2}, \sqrt{\frac{\mu_{e} \pi}{2 \rho_{0}}} \frac{k_{1} H}{\in d}\right]$, violation of which does not necessarily imply the occurrence of overstability.

\section{Nomenclature}

$C_{0}$ and $C_{1}$ - concentration at the bottom surface at $z=0$ and upper surface at $z=d$

$c, p_{1}, p_{2}$ - speed of light, the Prandtl number, magnetic Prandtl number

$$
d \text { - fluid thickness }
$$

$\boldsymbol{g}(0,0,-g)$ - acceleration due to gravity

$\boldsymbol{H}(=H, 0,0) \quad-$ magnetic field

$k\left(=\sqrt{k_{x}^{2}+k_{y}^{2}}\right)$ and $n-$ resultant wave number and growth rate, which is in general a complex constant

$N, e$ and $q$ - electron number density and change of an electron and Schmidt number.

$P_{l}$, and $F$ - dimensionless medium permeability and dimensionless couple-stress parameter

$R_{l}, S_{1}, Q_{1}, P$ and $M$ - Rayleigh number, stable solute gradient, magnetic field, medium permeability and Hall currents parameters

$\bar{R}_{c}$ and $R_{c}$ - critical Rayleigh number in the presence and absence of compressibility

$T_{0}$ and $T_{1}$ - temperature at the bottom surface $z=0$ and upper surface at $z=d$

$\delta p$, and $\delta \rho-$ perturbation in pressure and density

$\epsilon$, and $k_{l}$ - medium porosity and medium permeability

$\theta$, and $\gamma$ - perturbation in temperature $T$ and concentration $C$

$\kappa, \alpha$, and $\eta-$ thermal diffusivity, coefficient of thermal expansion and electrically resistivity

$\mu_{e}, \beta$, and $\beta^{\prime}$ - magnetic permeability, magnitudes of uniform temperature and concentration gradients $\zeta$ and $\xi-z$ - components of the vorticity and Hall current density

\section{Acknowledgement}

The author is grateful to the referees for their technical comments and valuable suggestions, resulting in a significant improvement of the paper.

\section{References}

[1] Joseph D.D. (1976): Stability of fluid motions. - Berlin: Springer-Verlag, vol.1 and 2.

[2] Bunch T.W., Hunder G.G. and Offord K. et. al. (1974): Synovial fluid complement: usefulness in diagnosis and classification of rheumatoid arthritis. - Ann. Intern. Med., vol.81, 32.

[3] Cohen A.S., Brandt K.D. and Krey P.K. (1975): Synovial fluid in laboratory diagnostic procedures. - In Cohen, A.S. (ed): The Rheumatic Diseases, $2^{\text {nd }}$ ed. Boston, Little, Brown and Co..

[4] Stokes V.K. (1966): Couple-Stresses in fluids. - Phys. Fluids, vol.9, pp.1709-1715.

[5] Stokes V.K. (1984): Theories of Fluids with Miscrostructure. - New York: Springer-Verlag.

[6] Walicki E. and Walicka A. (1999): Inertia effect in the squeeze film of a couple-stress fluid in biological bearings. Appl. Mech. Engng., vol.2, pp.363-373. 
[7] Sunil, Sharma Y.D., Bhart P.K. and Sharma R.C. (2005): Thermosolutal instability of compressible Rivlin-Ericksen fluid with Hall currents. - International J. of Applied Mechanics and Engineering, vol.10, No.2, pp.329-343.

[8] Malashety M.S. and Kollur P. (2011): The onset of double-diffusive convection in a couple-stress fluid saturated anisotropic porous layer. - Transp. Porous Med., vol.86, pp.435-459.

[9] Malashety M.S., Dulala P. and Premila K. (2010): Double-diffusive convection in a Darcy porous medium saturated with couple-stress fluid. - Fluid Dyn. Res., vol.42, pp.35502-35523.

[10] Malashety M.S., Gaikwad S.N. and Swamy M. (): An analytical study of linear and non-linear double-diffusive convection with Soret effect in couple-stress liquids. - Int. J. Them. Sci., vol.45, No.9, pp.897-907.

[11] Singh M. and Kumar P. (2009): Rotatory thermosolutal convection in a couple - stress fluids. - Z. Naturforch 64a, pp.448-454.

[12] Singh M. and Kumar P. (2012): Thermosolutal convection in a couple-stress fluid in uniform vertical magnetic field. - Z. Naturforch, vol.67a, pp.275-281

[13] Singh M. and Kumar P. (2011): Magneto and rotatory thermosolutal convection in couple-stress fluid in porous medium. - Journal of Porous Medium (Germany), vol.14, No.7, pp.637-648.

[14] Sharma R.C. and Rani N. (1988): Hall effect on thermosolutal instability of a plasma. - Int. J. of Pure and Applied Mathematics, vol.19, No.2, pp.202-207.

[15] Sunil, Sharma R.C. and Chandel R.S. (2004): Effect of suspended particles on couple-stress fluid heated and soluted from below in porous medium. - J. Porous Media, vol.7, No.1, pp.9-18.

[16] Singh M. (2011): Hall current effect on thermosolutal instability in viscoelastic fluids through porous medium. IJAME, vol.16, No.1, pp.69-82.

[17] Bishnoi J., Jawla V. and Kumar V. (2013): Thermal convection in a couple-stress fluid in the presence of horizontal magnetic field with Hall currents. - AAM, vol.8, No.1, pp.161-177.

[18] Veronis G. (1965): On finite amplitude instability in thermohaline convection. - J. Marine Res, vol.23, pp.1-17.

Received: July 23, 2017

Revised: June 6, 2018 